die Aufgaben von Akkreditierungsrat und Akkreditierungsagenturen und grenzt diese insbesondere gegeneinander ab. Die Akkreditierungsagenturen bereiten in Unterstützung durch Gutachterteams Akkreditierungsentscheidungen vor; der eigentliche Rechtsakt (Verleihung oder Versagung der Akkreditierung) wird dabei jedoch durch den Akkreditierungsrat vorgenommen.

Der Staatsvertrag wurde in Folge eines Beschlusses des Bundesverfassungsgerichts vom 17. Februar 2016 geschlossen.

\section{Die Rolle von DMV, GAMM und KMathF}

DMV, GAMM und KMathF können versuchen, durch Arbeitspapiere Einfluss auf die Ausgestaltung der Rechtsverordnungen der Länder zu nehmen. Außerdem können Sie Handlungsspielräume in Akkreditierungsverfahren aufzeigen und einen Diskussionsrahmen bieten. In diesem Sinne wollen wir "fachbezogene Referenzsysteme“ verstehen. Solche Arbeitspapiere dürfen aber niemals verbindlichen Charakter gewinnen, der Rechtsverordnungen der Länder vergleichbar wäre. Auch diese müssen gemäß dem oben erwähnten Beschluss die grundgesetzlich garantierte Wissenschaftsfreiheit respektieren.

Die DMV hat sich diesbezüglich in Denkanstößen (Mitteilungen 17 (2009), 239-242) eindeutig positioniert; dieses Papier ist Beschlusslage der DMV und wurde der KMathF zugeleitet, diese hat das Papier allerdings nie verabschiedet:
Die DMV und KMathF vertreten mit Nachdruck die Ansicht, dass Fakultäten, Hochschulen, Akkreditierungsrat und Akkreditierungsagenturen gemeinsam die durch die Rahmenvorgaben gegebenen Spielräume nutzen und die Festlegung von verbindlichen Regeln auf das erforderliche Mindestmaß beschränken sollen.

Akkreditierungsverfahren sollten grundsätzlich davon ausgehen, dass die Mehrzahl der Studiengänge seriös strukturiert ist, und einfache Verfahren entwickeln, eventuelle Missstände aufzudecken und abzustellen.

Die DMV und KMathF vertreten mit Nachdruck die Ansicht, dass es nicht angehen kann, dass ein erheblicher Anteil der Zeit- und Mittelressourcen der ohnehin überlasteten Hochschulen durch überzogene Akkreditierungsverfahren gebunden werden. Qualitätssicherung ist wichtig, muss aber einfach und effizient implementiert werden.

Auch wenn es mittlerweile eine Reihe von Regelwerken im Rahmen von Akkreditierungen zu beachten gilt, haben die Fächer in der Regel noch einen recht großen Gestaltungsspielraum. Ohne Not sollten die Fächer sich diesen Spielraum nicht einschränken lassen.

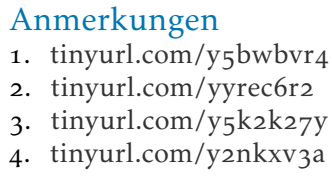

3. tinyurl.com/y5 $2 \mathrm{k} 27 \mathrm{y}$

4. tinyurl.com/y2nkxvza

\title{
Einladung zur Mitgliederversammlung der DMV
}

\author{
Karlsruhe, 24.9.2019
}

Im Auftrag des Präsidenten der Deutschen MathematikerVereinigung, Friedrich Götze, lade ich alle DMV-Mitglieder herzlich ein, an unserer Mitgliederversammlung während der Jahrestagung der DMV in Karlsruhe (23.26. 9. 2019) teilzunehmen.

Die Mitgliederversammlung der DMV findet am Dienstag, dem 24. 9. 2019, von 17-18 Uhr im Seminarraum 1.067, Englerstraße 2, Gebäude 20.30, Karlsruher Institut für Technologie, 76131 Karlsruhe, statt.

\author{
Tagesordnung \\ TOP 1: Genehmigung der Tagesordnung \\ TOP 2: Bericht des Präsidenten \\ TOP 3: Format der Jahrestagung \\ TOP 4: Minkowski-Medaille \\ TOP 5: Diskussion in der Mitgliederversammlung \\ TOP 6: Kassenbericht, Bericht der Kassenprüfer, \\ Entlastung des Vorstands \\ TOP 7: Wahl der Kassenprüfer \\ TOP 8: Anstehende Jahrestagungen \\ TOP 9: Verschiedenes
}

Informationen zu TOP 4 finden Sie im Grußwort dieses Heftes auf S. 57. Ich freue mich darauf, Sie in Karlsruhe zu begrüßen. Daniel Grieser (Schriftführer der DMV) 